\title{
MODELAGEM DOS DADOS DE FALHA DE UM CAMINHÃO FORA DE ESTRADA $^{1}$
}

\author{
Vítor Justus Leal ${ }^{2}$ \\ Paulo César de Resende Andrade ${ }^{3}$
}

\begin{abstract}
RESUMO
O mercado cada vez mais competitivo, ocasionado pela globalização, impõe que as empresas tenham um compromisso ainda maior com a redução dos seus custos. Os clientes necessitam de equipamentos com alta disponibilidade e confiabilidade. $\mathrm{Na}$ mineração, um dos custos expressivos existentes refere-se a falhas nos caminhões fora de estrada. Baseado na representatividade dos custos destas falhas, faz-se necessária a aplicação de técnicas de confiabilidade com a finalidade de aumentar a vida útil e o desempenho destes ativos. $\mathrm{O}$ presente trabalho tem por objetivo modelar o tempo de vida em horas de um caminhão fora de estrada utilizado na mineração, por meio da análise de confiabilidade paramétrica. $O$ procedimento de pesquisa utilizado foi o estudo de caso. Foram coletados dados de tempo até a falha de todos os sistemas do caminhão durante o período de um ano. Além dos métodos gráficos, foram utilizados os testes de aderência de Qui-Quadrado e Kolmogorov-Smirnov para verificar qual distribuição melhor se ajusta ao conjunto amostral. A análise foi feita com o software ProConf. Os parâmetros das distribuições foram determinados pelo método da máxima verossimilhança. Utilizou-se o software $\mathrm{R}$ para verificar o ajuste por meio do critério AIC. A distribuição Exponencial foi que melhor se ajustou ao conjunto de dados. A função de risco teve um comportamento constante, indicando que o caminhão está na fase de maturidade, em plena vida útil. A estratégia para esta fase é adotar a manutenção preditiva, realizando as manutenções e monitoramentos necessários para maximização da sua performance, com o intuito de evitar o início da fase de desgaste dos seus componentes.
\end{abstract}

Palavras-chave: Confiabilidade. Tempo de vida. Equipamento de grande porte.

\section{INTRODUÇÃO}

O ambiente de mudanças nos mais diversos setores da sociedade tem tornado cada vez mais complexo o desenvolvimento de equipamentos, produtos e sistemas (SILVA et al., 2017a), sendo de vital importância que tenham melhores desempenhos e custos competitivos. Desta forma, pode-se afirmar que o não funcionamento satisfatório de um equipamento

\footnotetext{
${ }^{1}$ Como citar este artigo: LEAL, Vítor Justus; ANDRADE, Paulo César de Resende. Modelagem dos dados de falha de um caminhão fora de estrada. ForScience: revista científica do IFMG, Formiga, v. 6, n. 3, e00500, jul./dez. 2018. DOI: 10.29069/forscience.2017v5n1.e500.

${ }_{2}^{2}$ Autor para correspondência: Paulo César de Resende Andrade: paulo.andrade@ict.ufvjm.edu.br
} 
implica na capacidade de produção de uma empresa, consequentemente, aumentando seus custos em geral e não garantindo uma boa qualidade ao produto.

Em um contexto no qual ocorrem cada vez mais rápidas mudanças significativas no mercado, a manutenção dos ativos de produção, antes vista como um mal necessário, passou a desempenhar papel estratégico e a ser considerada indispensável à produção (SANTOS; MOTTA; COLOSIMO, 2007). Tendo em vista o atendimento das necessidades do cliente, torna-se essencial a criação de estratégias de manutenção e confiabilidade dos equipamentos a fim de evitar indisponibilidades e desperdícios gerados através de paradas por quebras, falhas ou produção de produtos defeituosos que acarretam no comprometimento dos resultados almejados pela organização (GREGOL; ANDRADE, 2014).

É por meio do conhecimento das vulnerabilidades e problemas dos produtos durante sua etapa de vida útil que se obtém informações de falhas, que quando utilizadas junto a técnicas estatísticas de forma correta, permitem à engenharia da confiabilidade estimar o tempo que um produto funcionará de forma continua sem falhas (BRAILE; ANDRADE, 2013). Neste contexto, surge uma intensa procura por equipamentos capazes de manter sua disponibilidade com mínimas probabilidades de falhas, resultando em bons investimentos a um menor custo de manutenção.

Dessa maneira, o estudo da confiabilidade é de suma importância, pois garante a comercialização de produtos confiáveis, visto que o cliente não espera falhas durante o tempo de uso dos mesmos. A análise destas falhas é fundamental para compreensão do tipo de manutenção mais adequada ao comportamento de desempenho dos equipamentos, evitando a aplicação de atividades desnecessárias ou ineficazes que reduzem a disponibilidade, confiabilidade e geram custos operacionais (MENDES, 2011).

A alta competitividade entre os produtores de minério no mercado atual leva a melhorias no processo produtivo, como reduções de custos, aumento da produção, da qualidade e priorização da segurança.

A mineração constitui uma atividade dispendiosa, além de envolver alto potencial de riscos, mas, se conduzida de maneira adequada, sua indústria pode gerar ganhos consideráveis. Estima-se que o setor de transporte, na mineração, pode contribuir em até $40 \%$ dos custos totais da produção, dependendo do método de lavra adotado. Sendo assim, a implantação de uma melhoria neste âmbito implica em significativo impacto no custo final de produção, bem como nos lucros rendidos.

Neste contexto, uma redução e um controle do tempo de falha do caminhão, mantendo-se ou elevando-se a produção, gera não apenas ganhos financeiros para a empresa 
(com a redução de custos), mas também ambientais. O foco do trabalho constitui um dos principais gargalos referentes ao custo produtivo na atual cadeia de produção mineral. Especificamente, a abordagem aqui descrita é relativa à frota de transporte de materiais de lavra de mina, composta por caminhões fora de estrada.

A proposta deste artigo é modelar um estudo de caso aplicado em um caminhão fora de estrada de uma mineradora multinacional em uma de suas sedes situada no sudeste do Pará. Serão analisados os dados de falha levantados no ano de 2016, tendo como critério o tempo entre as falhas que ocorreram para manutenção corretiva.

O papel do caminhão dentro da empresa é imprescindível para que não prejudique a produção e há a necessidade de criação e implantação de técnicas de monitoramento e controle que proporcionem a diminuição de perdas através de uma detecção rápida de problemas, seguida da tomada de medidas corretivas.

\section{REFERENCIAL TEÓRICO}

\subsection{Confiabilidade}

Conforme Fogliatto e Ribeiro (2009), a confiabilidade de um item corresponde à sua probabilidade de desempenhar adequadamente o seu propósito, por um determinado período de tempo e sob condições predeterminadas. Já Haviaras (2005) define confiabilidade como a possibilidade de um componente ou equipamento executar a sua função sob condições de operação, por um período de tempo pré-determinado, sem apresentar falhas.

As funções mais utilizadas para análise da confiabilidade são as funções de confiabilidade e de risco. A função de confiabilidade $R(t)$ é representada em termos de uma probabilidade, pois ela nada mais é que a probabilidade de um sistema cumprir uma missão preestabelecida, com uma duração determinada, sem falhas. A taxa de risco ou taxa de falha $h(t)$ é considerada como sendo a quantidade de risco relacionada a uma unidade do tempo $t$, consequentemente sendo dada em termos de falha por unidade de tempo. O tempo médio até a falha (Mean Time To Failure - MTTF) define uma média de todos os tempos mensurados até ocorrer uma falha na unidade observada em questão (LEEMIS, 1995).

A análise possibilita, por meio de estimativa, caracterizar os comportamentos da confiabilidade, da probabilidade de falha e da taxa de falha em relação ao tempo de um componente, equipamento ou sistema (SANTOS et al., 2017). Para isso, é necessário fazer a modelagem de dados históricos de tempos entre falhas em distribuições de probabilidades. As 
distribuições de probabilidade que frequentemente são mais utilizadas para descrever os tempos até a falha de componentes e sistemas são: Weibull, Lognormal, Gama e Exponencial (HAVIARAS, 2005).

A distribuição de Weibull é uma das mais importantes distribuições em modelos de confiabilidade devido à flexibilidade e capacidade de representação de amostras de tempos até a falha com comportamentos distintos (LAFRAIA, 2001; FOGLIATTO; RIBEIRO, 2009; LEWIS, 1996). A distribuição Exponencial é relevante em estudos de confiabilidade por ser a única distribuição contínua em função do risco constante. A Lognormal é uma distribuição muito utilizada na modelagem de tempos até reparo em unidades reparáveis (FOGLIATTO; RIBEIRO, 2009).

A modelagem dos tempos até a falha é, portanto, central em estudos de confiabilidade (FOGLIATTO; RIBEIRO, 2009). Vários estudos foram desenvolvidos com comprovadas adequações a várias situações práticas (DUEK, 2005; BRANDÃO; ANDRADE, 2018, SANTOS et al., 2017; SILVA et al., 2017b, SILVA; ANDRADE, 2018).

\subsection{Confiabilidade e manutenção}

O comportamento da taxa de falha de um equipamento ao longo do tempo pode ser analisado pela curva da banheira, que representa genericamente a função de risco $h(t)$ ao longo do ciclo de vida.

A curva da banheira, representada na FIG. 1, apresenta três períodos característicos de vida de componentes e equipamentos: mortalidade infantil, fase de maturidade, e mortalidade senil (LAFRAIA, 2001; SELLITTO, 2005).

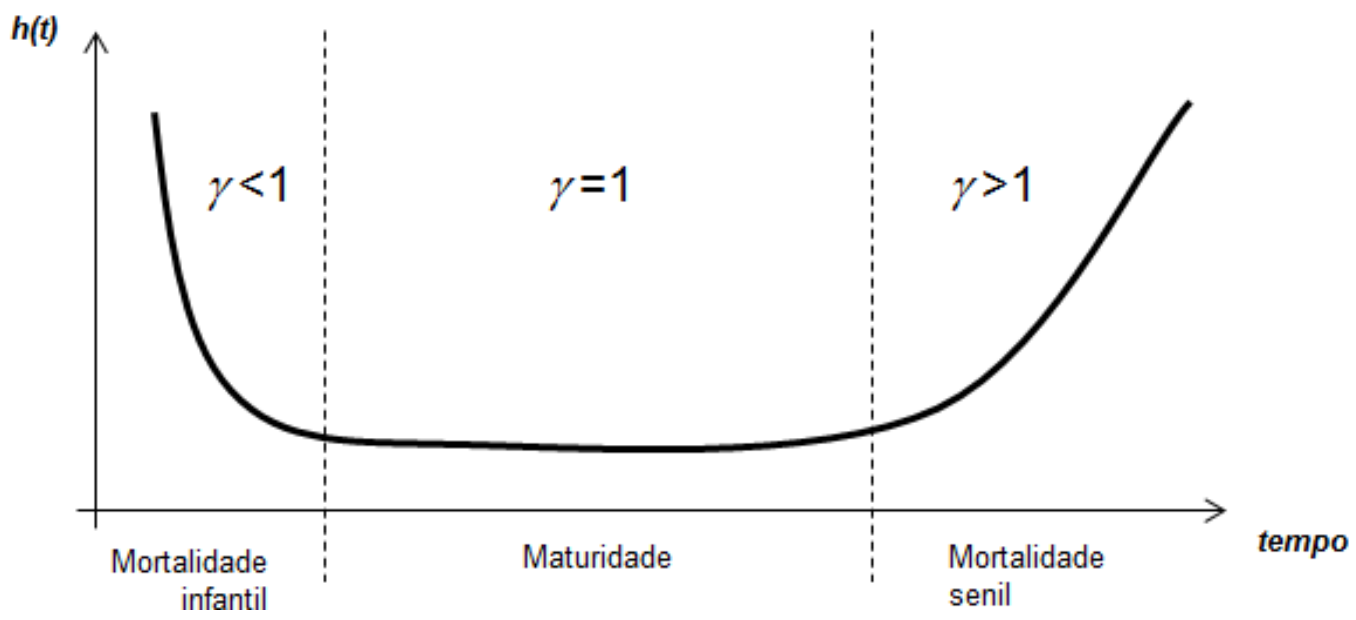

Figura 1 - Curva da banheira e o ciclo de vida dos equipamentos Fonte: Adaptado de Lafraia (2001) e Sellitto (2005). 
Sellitto (2005) relaciona cada fase da curva a um comportamento da função de risco $h(t)$ pelo parâmetro de forma $\gamma$ da distribuição de Weibull. O autor também associa a cada fase da curva uma estratégia de manutenção mais adequada sendo que no período inicial, chamado de mortalidade infantil, a taxa de falhas é alta, porém decrescente.

A estratégia para esta fase é a manutenção corretiva, que identifica e corrige deficiências de projeto ou de instalação do equipamento. No período seguinte, fase de maturidade, a taxa de falhas é sensivelmente menor e oscila ao redor de uma média constante. A estratégia para esta fase é a manutenção preditiva. O último período da curva é chamado de desgaste por Lafraia (2001) e de mortalidade senil por Sellitto (2005). Segundo os autores, é o fim da vida útil do equipamento e, neste período, a taxa de falhas é crescente, sendo a estratégia para esta fase é a manutenção preventiva: a troca antecipa a inevitável quebra.

\subsection{Caminhão fora de estrada}

Em minas de grandes extensões, a adoção de caminhões fora de estrada é usual devido à facilidade de realocação ante as diversas frentes de lavra em avanço, sendo ideal para terrenos adversos - como no caso da mineração - onde seria inviável a utilização de caminhões caçambas convencionais. Além disso, grandes projetos operam caminhões com capacidade de até 400 toneladas, otimizando o custo unitário de transporte do material.

Caminhões fora de estrada são utilizados em minas a céu aberto e subterrâneas, para transporte de material sólido a pequenas e médias distâncias. Além da evidente vantagem da flexibilidade em sua utilização, eles proporcionam ainda possibilidade de ciclos rápidos e vida útil de média a longa. Em contrapartida, a desvantagem é a manutenção onerosa, que torna extremamente importante o investimento mais amplo em um programa de manutenção preventiva eficiente, de modo a reduzir o número de paradas corretivas e perdas de produtividade. Por ser um trabalho que exige o máximo do equipamento, no caso transportar toneladas de minério em condições de terreno irregular, os números de falhas nestes caminhões são altos.

Ribeiro e Almeida (2014) fizeram estudo de confiabilidade de motores diesel de caminhões fora de estrada e Campos Júnior et al. (2013) desenvolveram uma metodologia para monitoramento e controle do consumo específico de diesel em caminhões fora de estrada. Silva et al. (2017b) estudaram os principais modos de falha dos pneus deste tipo de caminhão, propondo ações de melhorias no intuito de otimizar sua performance e após a 
implantação destas ações, observou-se uma melhora significativa nos indicadores de gestão dos pneus, levando a conclusão dos impactos positivos que o projeto trouxe a mineração.

\section{MATERIAL E MÉTODOS}

A pesquisa empregada foi o estudo de caso de um caminhão fora de estrada. Os dados em questão foram de uma das minas da Vale, na região de Carajás, em Parauapebas/PA, na qual a empresa se destaca por ser uma das maiores mineradoras do mundo e a primeira colocada na produção mundial de minério de ferro, pelotas e níquel. Carajás possui a maior frota de equipamentos de mina da Vale sendo 147 caminhões fora de estrada. Devido à grande extração de minério realizada pela empresa, é necessário que tenha uma frota de caminhões de grande porte para que a produção não pare.

A função do caminhão dentro da mina é fazer o transporte do minério lavrado até a usina de beneficiamento, onde este será processado conforme expedição do cliente. $\mathrm{O}$ caminhão analisado é do modelo CATERPILLAR 777G, com peso bruto total de 164,6 toneladas, capacidade da caçamba é de $64,3 \mathrm{~m}^{3}$, podendo carregar no máximo 99,8 toneladas, sendo que sua velocidade máxima carregado é de $67 \mathrm{~km} / \mathrm{h}$.

A empresa disponibilizou os dados de falhas de 40 caminhões em operação, todos do mesmo modelo, fabricante e capacidade de carga. Os sistemas analisados foram transmissão, elevação, motor, locomoção, ar condicionado, frenagem, elétrico, cabine, direção e pneumático. Para o estudo estatístico deste artigo foram utilizados os tempos até a falha do caminhão com maior número de ocorrências na manutenção durante o ano de 2016. Esse período representa o início e fim de um período completo de funcionamento até a falha. A TAB. 1 mostra os tempos até falha coletados.

Tabela 1 - Tempo até a falha de um caminhão fora de estrada

\begin{tabular}{|c|c|c|c|c|c|c|c|}
\hline $\mathrm{N}$ & $\begin{array}{c}\text { Tempo até } \\
\text { a falha }\end{array}$ & $\mathrm{n}$ & $\begin{array}{c}\text { Tempo até } \\
\text { a falha }\end{array}$ & $\mathrm{n}$ & $\begin{array}{c}\text { Tempo até } \\
\text { a falha }\end{array}$ & $\mathrm{n}$ & $\begin{array}{c}\text { Tempo até } \\
\text { a falha }\end{array}$ \\
\hline 1 & 224,4 & 15 & 325,5 & 29 & 23,1 & 43 & 22,5 \\
\hline 2 & 42,0 & 16 & 209,0 & 30 & 24,2 & 44 & 61,1 \\
\hline 3 & 112,2 & 17 & 145,3 & 31 & 180,5 & 45 & 167,3 \\
\hline 4 & 3,6 & 18 & 258,1 & 32 & 89,0 & 46 & 208,5 \\
\hline
\end{tabular}




\begin{tabular}{rrrrrrrr}
\hline 5 & 89,0 & 19 & 507,3 & 33 & 131,3 & 47 & 24,5 \\
6 & 30,1 & 20 & 165,3 & 34 & 47,3 & 48 & 8,4 \\
7 & 185,0 & 21 & 22,0 & 35 & 24,0 & 49 & 22,0 \\
8 & 66,0 & 22 & 776,0 & 36 & 182,2 & 50 & 62,5 \\
9 & 144,0 & 23 & 30,1 & 37 & 22,3 & 51 & 32,4 \\
10 & 74,0 & 24 & 140,4 & 38 & 232,5 & 52 & 75,2 \\
11 & 5,3 & 25 & 168,0 & 39 & 29,0 & 53 & 38,0 \\
12 & 328,0 & 26 & 250,1 & 40 & 23,4 & 54 & 24,3 \\
13 & 235,5 & 27 & 102,7 & 41 & 92,3 & 55 & 92,1 \\
14 & 39,3 & 28 & 258,5 & 42 & 349,5 & 56 & 86,5 \\
\hline Fonte: Vale S/A. & & & & & & (Conclusão.)
\end{tabular}

Os dados disponibilizados foram trabalhados no ProConf (FRITSCH; RIBEIRO, 1998), um programa computacional projetado para o ajuste de distribuições de tempos de falha para dados de confiabilidade, por meio de métodos gráficos e métodos analíticos. Os histogramas de frequência das falhas e os papéis de probabilidades foram utilizados como métodos gráficos para comparar as curvas das distribuições de probabilidade e verificar o modelo que apresenta melhor aderência aos dados amostrais.

Para a caracterização da distribuição de frequência foram aplicados testes de aderência às distribuições de probabilidades. Os testes utilizados para verificar o ajuste destas distribuições candidatas aos dados efluentes foram: Qui-Quadrado $\left(\chi^{2}\right)$ e KolmogorovSmirnov (K-S). O software informa o nível de significância e aponta quais distribuições não podem ser rejeitadas (FRITSCH; RIBEIRO, 1998). A validação é dada se o nível de significância for maior que 5\% em ambos os testes de aderência. Quando o mesmo é maior a 5\% em ambos os testes, do Qui-Quadrado e do Kolmogorov-Smirnov, a distribuição não pode ser rejeitada, o que significa que poderá ser utilizada na modelagem. Caso mais do que uma distribuição não possa ser rejeitada, cabe ao pesquisador justificar a escolha por uma delas por fundamentação teórica.

Para verificar a adequação da validação do desempenho dos modelos foi utilizado o Critério de Informação de Akaike - Akaike's Information Criterion (AIC). Quanto menor for o valor de AIC, melhor será o ajuste do modelo (AKAIKE, 1973). Para tal foi utilizado o software R (R FOUNDATION, 2018).

Em seguida, são fornecidas as estimativas dos parâmetros da distribuição de probabilidade que melhor modela o conjunto de dados em estudo, utilizando o método de 
máxima verossimilhança. Além disso, são apresentadas as representações das funções de confiabilidade $R(t)$ e de risco ou taxa de falha $h(t)$.

\section{RESULTADOS E DISCUSSÕES}

Inicialmente, foram obtidos alguns gráficos relativos ao conjunto de dados. Na FIG. 2 está representado o histograma dos tempos de falhas apresentados na TAB. 1. O formato do histograma sugere a aplicação das distribuições, Weibull, Exponencial e Lognormal para modelar adequadamente o conjunto de dados estudados.

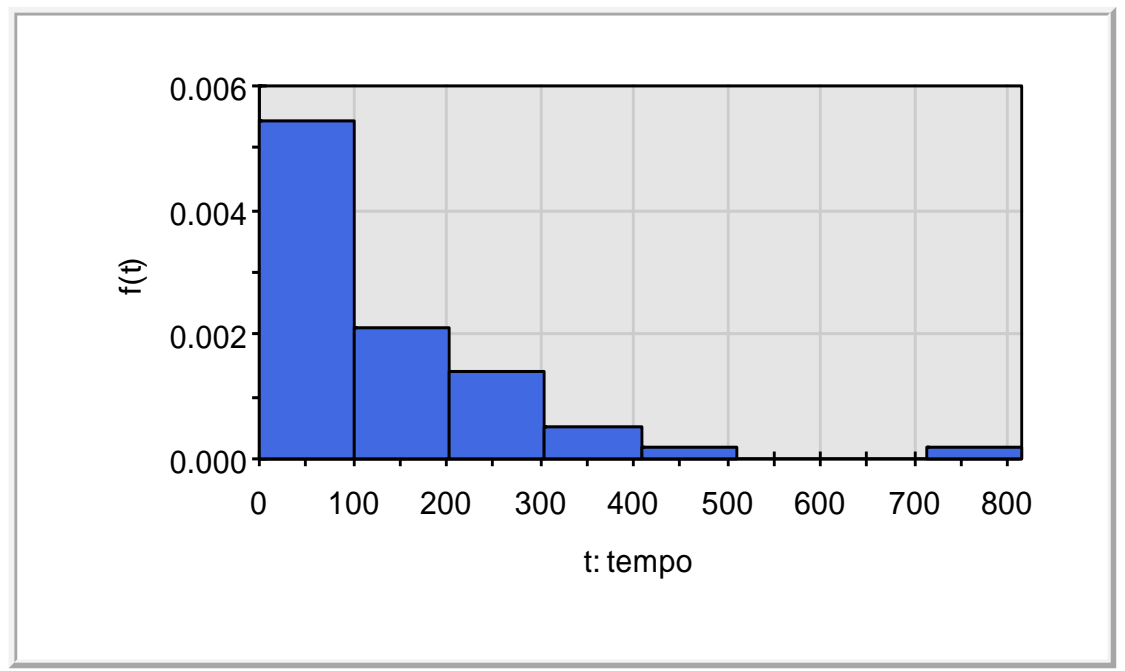

Figura 2 - Gráfico de frequência das falhas Fonte: ProConf.

A análise dos papéis de probabilidade, mostradas na FIG. 3, indicam que nenhuma das distribuições apresentam uma linha de tendência, ou seja, apresentam desvios, não se ajustando perfeitamente em torno da reta. Desta forma, não é possível definir o modelo que melhor se ajusta ao conjunto amostral. 

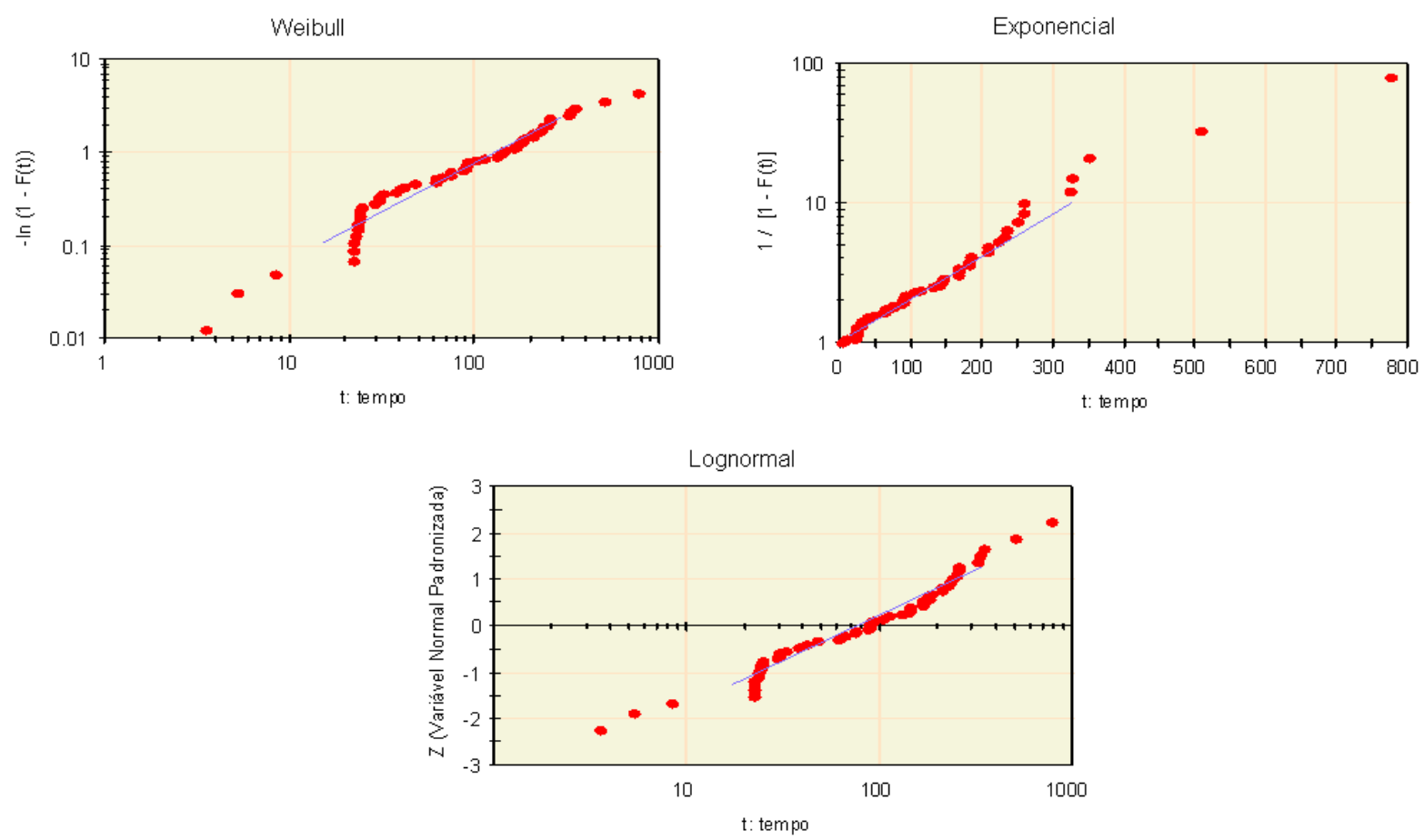

Figura 3 - Papel de probabilidade das distribuições Weibull, Exponencial e Lognormal. Fonte: ProConf.

As significâncias dos testes de aderência Qui-Quadrado $\left(\chi^{2}\right)$ e de Kolmogorov-Smirnov (K-S) são apresentadas na Tabela 2. No teste do Qui-Quadrado $\left(\chi^{2}\right)$ verifica-se que o nível de significância é maior para a Exponencial se comparado as demais distribuições. No teste de Kolmogorov-Smirnov (K-S) a maior significância foi obtida para a distribuição Lognormal. Esses resultados corroboram com a análise anterior, feita nos papéis de probabilidade.

Tabela 2 - Níveis de significância dos testes de aderência

\begin{tabular}{cccc}
\hline Modelo & $\chi^{2}$ & K-S & Decisão \\
\hline Weibull & 0,5757 & 0,1204 & Não pode ser rejeitada \\
Exponencial & 0,6623 & 0,1532 & Não pode ser rejeitada \\
Lognormal & 0,5010 & 0,1915 & Não pode ser rejeitada \\
\hline
\end{tabular}

Fonte: Dos autores, 2018.

Apesar de não haver rejeição de nenhuma das três distribuições, deve-se dar maior importância ao teste de aderência do Qui-Quadrado, pelo fato do tamanho da amostra ser maior que 30 (FOGLIATTO; RIBEIRO, 2009).

Para validação do desempenho dos modelos foi calculado o AIC, via software R. De acordo com este critério, quanto menor o valor do AIC melhor será o ajuste do modelo. Desta 
forma a hipótese de que a distribuição Exponencial é a mais indicada para se ajustar ao estudo de caso foi confirmada. Os valores estão apresentados na TAB. 3.

Tabela 3 - Valores do teste AIC

\begin{tabular}{cc}
\hline Modelo & AIC \\
\hline Weibull & 661,6453 \\
Exponencial & 659,6604 \\
Lognormal & 662,4557 \\
\hline
\end{tabular}

Fonte: Adaptado do software R.

Dessa forma pode-se afirmar que a distribuição Exponencial é a mais apropriada para o estudo da confiabilidade do caminhão fora de estrada em questão e seus parâmetros ajustados podem ser utilizados durante os estudos de confiabilidade, pois os resultados dos papéis de probabilidade e testes de aderência se ajustam melhor a essa distribuição. A TAB. 4 apresenta os parâmetros da distribuição de Exponencial, ajustados pelos dados experimentais da TAB. 1.

Tabela 4 - Resultados dos ajustes - Exponencial

\begin{tabular}{cc}
\hline Parâmetro & Resultados \\
\hline$t_{10}$ (horas) & 13,76 \\
$t_{50}$ (horas) & 90,51 \\
MTTF (horas) & 130,58 \\
$\lambda$ & 0,0077 \\
\hline
\end{tabular}

Fonte: Adaptado do software ProConf.

Pela TAB. 4, o tempo médio até a falha ocorrer (MTTF) no caminhão fora de estrada é 130,58 horas e a metade dos caminhões falhou antes de 91 horas. $\mathrm{O} t_{10}$ e $t_{50}$ correspondem aos valores limites dos tempos, nos quais $10 \%$ e $50 \%$ das falhas ocorreram.

Levando em consideração os resultados dos testes de aderência e do critério AIC, a distribuição Weibull apresentou resultados bem similares ao da Exponencial, conforme TAB. 5. 
Tabela 5 - Resultados dos ajustes - Weibull

\begin{tabular}{cc}
\hline Parâmetro & Resultado \\
\hline$t_{10}$ (horas) & 13,31 \\
$t_{50}$ (horas) & 89,63 \\
MTTF (horas) & 130,56 \\
$\gamma$ & 1,01 \\
$\theta$ & 131,29 \\
\hline
\end{tabular}

Fonte: Adaptado do software ProConf.

Isso se deve ao fato de que o valor do parâmetro gama $\gamma$ estar bem próximo de 1 . Quando $\gamma$ é igual a 1, a taxa de falha é constante e a Weibull possui o mesmo comportamento da distribuição Exponencial (LEE; WANG, 2003; LEEMIS, 1995).

Estabelecendo-se uma analogia do comportamento da taxa de falhas constante do modelo Exponencial, com a fase de maturidade da curva da banheira da distribuição Weibull, indica-se que a manutenção ideal seria a preditiva (SELLITTO, 2005). Esse tipo de manutenção é realizado conforme a necessidade, com base no resultado de inspeções contínuas ou periódicas. As falhas são casuais e decorrentes de fatores menos controláveis, tais como: mau uso do equipamento, ultrapassagem de resistência ou fenômenos naturais imprevisíveis. Quando o grau de degradação atinge o limite estabelecido, uma intervenção de manutenção é executada antes da falha (SELLITTO, 2005).

A função confiabilidade está representada na FIG. 4. Constata-se que a confiabilidade sempre se dá em uma curva decrescente em função do tempo, já que as probabilidades de perfeitas condições decaem conforme há a utilização e o desgaste do equipamento.

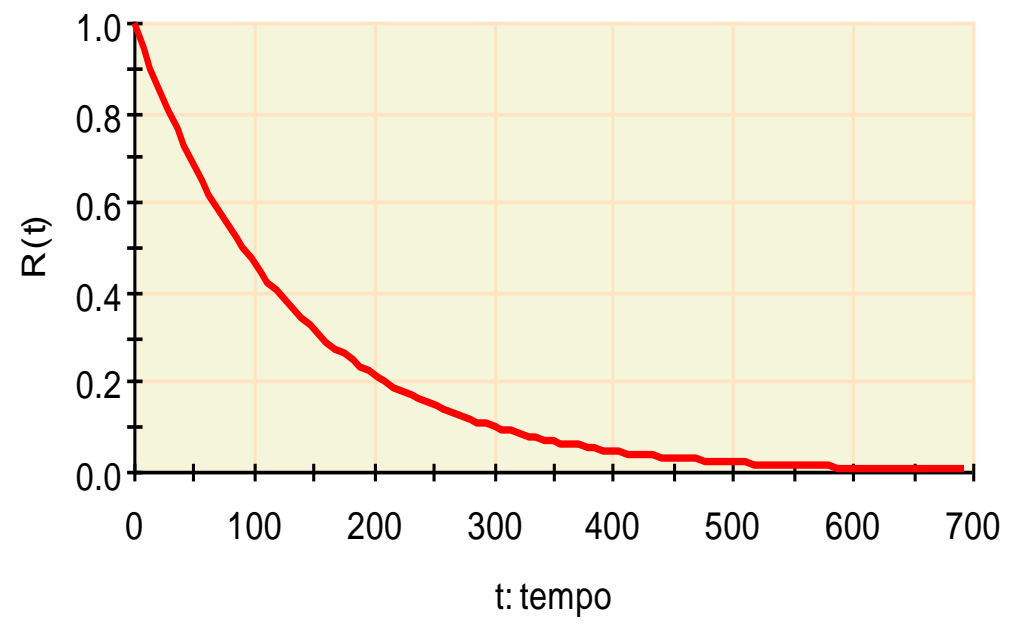

Figura 4 - Função confiabilidade

Fonte: ProConf 
A função de risco está representada na FIG. 5.

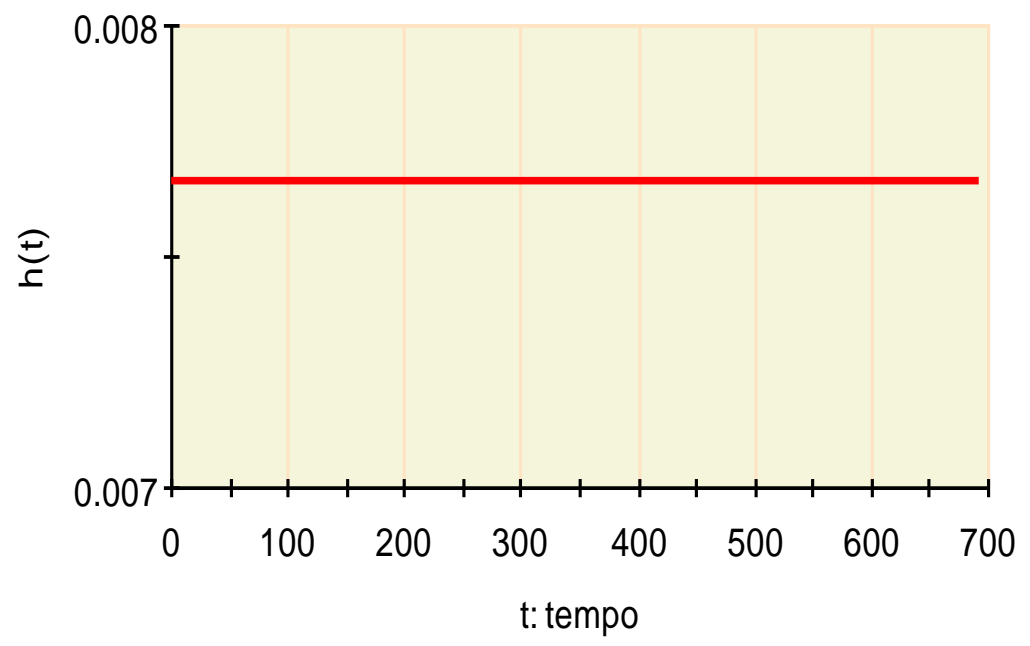

Figura 5 - Função taxa de falha Fonte: ProConf.

Nota-se que o equipamento mantém uma taxa de falha relativamente constante na maioria do tempo decorrido, ou seja, a função de risco do modelo Exponencial é constante.

Como o modelo Exponencial foi adequado na modelagem, sabe-se que o risco de falha para as unidades que estão em estudo há pouco ou muito tempo, dado que ainda não tenham falhado, é o mesmo (LEE; WANG, 2003). Essa propriedade é conhecida como a falta de memória.

\section{CONCLUSÕES}

Muitos estudos estatísticos de manutenção e confiabilidade utilizam dados simulados de horas até a falha ou são realizados submetendo os equipamentos a um regime de estresse para estimar sua vida útil. Neste estudo foram utilizados dados reais com o equipamento em regime normal de trabalho, tornando possível verificar, através da análise estatística, que os dados corroboram para o uso de distribuições já conhecidas.

Os dados de tempo até a falha mostram que a distribuição Exponencial se adequa melhor aos dados. O valor do parâmetro forma da distribuição Weibull obtido mostra que o comportamento do caminhão fora de estrada se localiza na segunda fase da curva da banheira, indicativo de que a manutenção ideal para o equipamento em questão será a preditiva. Desta forma, os resultados encontrados permitem à empresa conhecer melhor o perfil das falhas dos caminhões fora de estrada de sua frota. 
Por fim conclui-se que a confiabilidade é de suma importância dentro de um processo industrial, pois o resultado obtido permite que a empresa reduza custos com mão de obra, não perca tempo de produção com a substituição de equipamentos, possibilitando um melhor funcionamento da empresa de forma geral. As análises quantitativas dos tempos até as falhas são relevantes para a determinação de estratégias de manutenção a ser realizadas pelas empresas, a fim de garantir maior disponibilidade dos produtos.

\title{
MODELING THE FAILURE DATA OF AN OFF-ROAD TRUCK
}

\begin{abstract}
The increasingly competitive market, caused by globalization, requires that companies have an even greater commitment to reduce their costs. The customers need equipment with high availability and reliability. In mining, one of the significant costs refers to failures in off-road trucks. Based on the representativeness of the costs of these failures, it is necessary to apply reliability techniques with the purpose of increasing the useful life and performance of these assets. The present work aims to model the time of life, in hours, of an off-road truck used in mining, through parametric reliability analysis. The research procedure used was the case study. Time data were collected until failure of all truck systems, resulting in fifty-six failures during the period of one year. Besides the graphical methods, the adhesion tests of Chi-square and Kolmogorov-Smirnov were used to verify which distribution best fits the sample set. The analysis was done with ProConf software. The parameters of the distributions were determined by the maximum likelihood method. The software $\mathrm{R}$ was used to verify the adjustment using the AIC criterion. The Exponential distribution was the best fit to the data set. The risk function had a constant behavior, indicating that the truck is in the phase of maturity, in full working life. The strategy for this phase is to adopt the predictive maintenance, performing the necessary maintenance and monitoring to maximize its performance, in order to avoid the beginning of the wear phase of its components.
\end{abstract}

Keywords: Reliability. Lifetime. Large equipment.

\section{REFERÊNCIAS}

AKAIKE, Hirotugu. Information Theory and an Extension of the Maximum Likelihood Principle. In: PARZEN, E.; TANABE, K.; KITAGAWA, G. (ed.). Selected papers of Hirotugu Akaike. Springer Series in Statistics (Perspectives in Statistics). New York, NY: Springer, 1998.

BRAILE, Nathalia Ávila; ANDRADE, Jairo José de Oliveira. Estudo de falhas em equipamentos de costura industriais utilizando o FMEA e a análise de confiabilidade. In: ENCONTRO NACIONAL DE ENGENHARIA DE PRODUÇÃO, 34, Salvador, 2013. Anais... Salvador, 2013. 
BRANDÃO, Mariane Olivier; ANDRADE, Paulo César de Resende. Modelagem dos dados de falhas de um pasteurizador de garrafas de cerveja. Revista de Engenharia e Tecnologia, v. 10, n. 2 , p. $172-181,2018$.

CAMPOS JÚNIOR, Carlos Roberto et al. Desenvolvimento de uma metodologia para redução do consumo específico de diesel em caminhões fora de estrada em uma empresa do setor de mineração. In: SIMPÓSIO DE EXCELÊNCIA EM GESTÃO E TECNOLOGIA, 10, Resende, 2013. Anais... Resende, 2013.

DUEK, Carlos. Análise de confiabilidade na manutenção de componente mecânico de aviação. 2005. 119 f. Dissertação (Mestrado em Engenharia de Produção) - Universidade Federal de Santa Maria, Santa Maria, 2005.

FOGLIATTO, Flávio Sanson; RIBEIRO, José Luis Duarte. Confiabilidade e manutenção industrial. Rio de Janeiro: Elsevier, 2009.

FRITSCH, Celso; RIBEIRO, José Luis Duarte. PROCONF: um software orientado para análises de confiabilidade. In: ENCONTRO NACIONAL DE ENGENHARIA DE PRODUÇÃO, 18, Niterói, 1998. Anais... Niterói, 1998.

GREGOL, Luciano Bernochi; ANDRADE, Jairo Jose de Oliveira. Análise de falhas como subsídio para o estabelecimento de procedimentos de manutenção produtiva total (MTP): estudo de caso de uma máquina gargalo na fabricação de latas de alumínio. In: ENCONTRO NACIONAL DE ENGENHARIA DE PRODUÇÃO - ENEGEP, 29, Curitiba, 2014. Anais... Curitiba, 2014.

HAVIARAS, Gilberto. J. Metodologia para análise de confiabilidade de pneus radiais em frota de caminhões de longa distância. 2009. 124 f. Dissertação (Mestrado em Engenharia Automotiva) - Escola Politécnica, Universidade de São Paulo, São Paulo, 2005.

LAFRAIA, João Ricardo Barusso. Manual de confiabilidade, mantenabilidade e disponibilidade. Rio de Janeiro: Qualitymark, 2001.

LEE, Elisa T.; WANG, John Wenyu. Statistical methods for survival data analysis. New Jersey: Wiley, 2003.

LEEMIS, L. M. Reliability: probabilistic models and statistical methods. New Jersey: Prentice-Hall, 1995.

LEWIS, E. Introduction to reliability engineering. New York: John Wiley \& Sons, 1996.

MENDES Angélica Alebrant. Manutenção centrada em confiabilidade: uma abordagem quantitativa. 2011. 85 p. Dissertação (Mestrado em Engenharia de Produção) - Escola de Engenharia, Universidade Federal do Rio Grande do Sul, Porto Alegre, 2011. 
RIBEIRO, Adriano Gonçalves dos Santos; ALMEIDA, Gean Carlo Feliciano. Estudo de confiabilidade de motores diesel de caminhões fora de estrada. Revista de Estatística da UFOP, Ouro Preto, v. 3, p. 460-464, 2014.

SANTOS, Wagner Baracho dos; MOTTA, Sergio Brandão da; COLOSIMO, Enrico Antônio. Tempo ótimo entre manutenções preventivas para sistemas sujeitos a mais de um tipo de evento aleatório. Revista Gestão e Produção, São Carlos, v. 14, n. 1, p. 193-202, 2007.

SANTOS, Maicon Mateus de Medeiros et al. Modelagem do tempo de vida de um inversor de frequência. ForScience: revista científica do IFMG, Formiga, v. 5, n. 3, e00288, 2017.

SELLITTO, M. Formulação estratégica da manutenção industrial com base na confiabilidade dos equipamentos. Produção, v.15, n.1, p.044-059, 2005.

SILVA, Evaldo da Conceição et al. Análise de Dados de Falha de um Transmissor de Fibra Óptica. Revista Thema, Pelotas, v. 14, n. 4, p. 259-266, 2017a.

SILVA, Elifas Levi da et al. Análise dos modos de falhas em pneus de caminhões fora de estrada em uma mineração. In: ABM ANNUAL CONGRESS, 72, São Paulo, 2017. Anais... São Paulo, 2017b.

SILVA, Ellen Maria Neves; ANDRADE, Paulo César de Resende. Análise de confiabilidade de um inspetor eletrônico de garrafas. Revista da Universidade Vale do Rio Verde, v. 16, n.2, p. 1-9, 2018.

THE R FOUNDATION. R: a language and environment for statistical computing. R Foundation for Statistical Computing, Vienna, Áustria, 2018.

\section{DADOS DOS AUTORES}

\section{Vítor Justus Leal}

E-mail: vitorjustus@gmail.com.

Currículo Lattes: http://lattes.cnpq.br/7816407877526299

Estudante de graduação em Ciência e Tecnologia pela Universidade Federal dos Vales do Jequitinhonha e Mucuri (UFVJM). Tem experiência na área de Ciência e Tecnologia, com ênfase em Engenharia Mecânica.

\section{Paulo César de Resende Andrade}

E-mail: pceandrade@gmail.com.

Currículo Lattes: http://lattes.cnpq.br/0894646446086485

Doutorado em Estatística e Experimentação Agropecuária, mestrado em Ciências (Agronomia - Estatística e Experimentação Agropecuária) pela Universidade Federal de Lavras (UFLA). Possui graduação em Engenharia Industrial Elétrica, graduação e especialização em Matemática. Atualmente é professor Associado II da Universidade Federal dos Vales do Jequitinhonha e Mucuri (UFVJM), Campus de Diamantina. 